\title{
Age-related magnetic susceptibility changes in deep grey matter and cerebral cortex of normal young and middle-aged adults depicted by whole brain analysis
}

\author{
Romana Burgetova $^{1,2}$, Petr Dusek ${ }^{1,3}$, Andrea Burgetova ${ }^{1}$, Adam Pudlac $^{1}$, Manuela Vaneckova ${ }^{1}$, \\ Dana Horakova ${ }^{3}$, Jan Krasensky ${ }^{1}$, Zsoka Varga ${ }^{3}$, Lukas Lambert ${ }^{1}$ \\ ${ }^{1}$ Department of Radiology, First Faculty of Medicine, Charles University and General University Hospital in Prague, Prague, Czech Republic; \\ ${ }^{2}$ Department of Radiology, Third Faculty of Medicine, Charles University and University Hospital Královské Vinohrady, Prague, Czech Republic; \\ ${ }^{3}$ Department of Neurology, First Faculty of Medicine, Charles University and General University Hospital in Prague, Prague, Czech Republic
}

Contributions: (I) Conception and design: P Dusek, A Burgetova, L Lambert; (II) Administrative support: A Burgetova, L Lambert, A Pudlac; (III) Provision of study materials or patients: D Horakova, Z Varga, P Dusek, M Vaneckova, J Krasensky; (IV) Collection and assembly of data: R Burgetova, M Vaneckova, P Dusek, J Krasensky, D Horakova; (V) Data analysis and interpretation: P Dusek, L Lambert, R Burgetova, A Burgetova; (VI) Manuscript writing: All authors; (VII) Final approval of manuscript: All authors.

Correspondence to: Assoc. prof. Andrea Burgetova, MD, PhD.First Faculty of Medicine Charles University and General Faculty Hospital in Prague, U nemocnice 2, 12808 Prague 2, Czech Republic. Email: andrea.burgetova@vfn.cz.

Background: Iron accumulates in brain tissue in healthy subjects during aging. Our goal was to conduct a detailed analysis of iron deposition patterns in the cerebral deep grey matter and cortex using region-based and whole-brain analyses of brain magnetic susceptibility.

Methods: Brain MRI was performed in 95 healthy individuals aged between 21 and 58 years on a $3 \mathrm{~T}$ scanner. MRI protocol included T1-weighted (T1W) magnetization-prepared rapid acquisition with gradient echo images and 3D flow-compensated multi-echo gradient-echo images for quantitative susceptibility mapping (QSM). In the region-based analysis, QSM and T1W images entered an automated multi-atlas segmentation pipeline and regional mean bulk susceptibility values were calculated. The whole-brain analysis included a non-linear transformation of QSM images to the standard MNI template. For the whole-brain analysis voxel-wise maps of linear regression slopes $\beta$ and $P$ values were calculated. Regional masks of cortical voxels with a significant association between susceptibility and age were created and further analyzed.

Results: In cortical regions, the highest increase of susceptibility values with age was found in areas involved in motor functions (precentral and postcentral areas, premotor cortex), in cognitive processing (prefrontal cortex, superior temporal gyrus, insula, precuneus), and visual processing (occipital gyri, cuneus, posterior cingulum, fusiform, calcarine and lingual gyrus). Thalamic susceptibility increased until the fourth decade and decreased thereafter with the exception of the pulvinar where susceptibility increase was observed throughout the adult lifespan. Deep grey matter structures with the highest increase of susceptibility values with age included the red nucleus, putamen, substantia nigra, dentate nucleus, external globus pallidus, caudate nucleus, and the subthalamic nucleus in decreasing order.

Conclusions: Accumulation of iron in basal ganglia follows a linear pattern whereas in the thalamus, pulvinar, precentral cortex, and precuneus, it follows a quadratic or exponential pattern. Age-related changes of iron content are different in the pulvinar and the rest of the thalamus as well as in internal and external globus pallidus. In the cortex, areas involved in motor and cognitive functions and visual processing show the highest iron increase with aging. We suggest that the departure from normal patterns of regional brain iron trajectories during aging may be helpful in the detection of subtle neurodegenerative and neuroinflammatory processes.

$\wedge$ ORCID: 0000-0002-9975-2338. 
Keywords: Magnetic susceptibility; iron; aging; brain; deep grey matter; cerebral cortex

Submitted Jan 23, 2021. Accepted for publication Apr 19, 2021.

doi: $10.21037 /$ qims-21-87

View this article at: http://dx.doi.org/10.21037/qims-21-87

\section{Introduction}

During the lifespan, the human brain undergoes microstructural changes including grey matter atrophy, myelin degeneration, or iron accumulation that can be picked in vivo by various quantitative MRI techniques (1-4). Knowledge about the spectrum of age-related changes in brain structure and associated MR parameters is important for understanding the neurobiology of aging and for differentiating physiological aging from disease.

Magnetic susceptibility, a measure that indicates the extent to which a material may be magnetized in external magnetic field, can be quantified in tissues by a recent MR technique called quantitative susceptibility mapping (QSM) (5). While magnetic susceptibility of cerebral tissue may be influenced by myelin, calcium, or deoxyhemoglobin, it is mainly determined by ferritin-iron content in the basal ganglia and other grey matter structures (6). QSM is thus considered as a surrogate measure of iron content in the grey matter $(7,8)$.

Cerebral accumulation of non-heme iron occurs during normal aging. This increase occurs heterogeneously in certain cell types and brain regions such as basal ganglia, hippocampus, motor cortex, cerebellar nuclei, and other subcortical brain regions (9-13). Globus pallidus, substantia nigra, red nuclei, and putamen are regions with the highest iron deposition (14-16).

Iron is involved in numerous pathways in the brain such as oxygen transportation, oxidative phosphorylation, DNA synthesis, mitochondrial respiration, myelin synthesis, antioxidant enzyme activation, and metabolism of neurotransmitters (13). On the other hand, disturbance of iron homeostasis can lead to brain neurotoxicity by different mechanisms such as membrane damage, protoxin bioactivation, aberrant cell signaling, bioenergetic failure, proteosomal and mitochondrial dysfunction, protein aggregation and inclusion formation, electrophysiological derangements, and synaptolysis, conveying all of these factors on apoptosis, necrosis or a specific cell death triggered by iron called ferroptosis $(13,17,18)$. Increased subcortical iron is associated with decreased cognitive and motor functions in the elderly population $(14,19,20)$ suggesting that basal ganglia may be more vulnerable to iron accumulation throughout aging, exposing them to an increased risk of neurodegeneration or/and neuroinflammation. Understanding normal patterns of iron accumulation with aging might thus assist in identifying subjects who are at greater risk of accelerated neurodegeneration.

The majority of previous studies examining iron accumulation during normal aging were based on region-ofinterest analysis of deep grey matter on R2* relaxometry and magnetic susceptibility maps (21-27). Whole-brain analysis, which is not limited to a priori predefined regions, has been used only sparsely for studying age-related changes of magnetic susceptibility and, by extension, of iron levels $(28,29)$.

The objective of this study was to conduct a detailed analysis of iron deposition patterns not only in the cerebral deep grey matter but also in the cortex by a voxel-wise analysis of QSM at the whole-brain level and using regionbased analysis.

\section{Methods}

The study was conducted in accordance with the Declaration of Helsinki (as revised in 2013). This study was approved by the Ethics Committee of the General University Hospital in Prague (ID1018/17), and all subjects signed informed consent.

\section{Study participants}

Brain MRI was performed in 95 healthy individuals (57 females and 38 males) aged between 21 and 58 (mean $\pm \mathrm{SD}=37 \pm 10$ ) years, who responded to an announcement presented at the First Faculty of Medicine, Charles University and General University Hospital in Prague and met the following inclusion criteria: (I) willing to sign an informed consent in accordance with local regulations, (II) age 18-60 years (chosen to be comparable to that of adult patients with multiple sclerosis). The exclusion criteria were: (I) unable to undergo MRI examination, (II) unable to be examined 4 times during the next three years at $0,12,24$, 
36 months, (III) pregnancy at the time of enrollment into the study, (IV) any diseases, medical conditions, or brain MRI abnormalities that could influence the structure of the brain or spinal cord.

\section{Imaging protocol}

The examination was performed on a 3T MRI scanner (Siemens Skyra 3T, Siemens Healthcare, Erlangen, Germany) with a 32-channel birdcage head coil. MRI protocol included T1-weighted 3D Magnetization-Prepared Rapid Acquisition with Gradient Echo (MPRAGE) images (T1WI) in sagittal plane with the following acquisition parameters: repetition time (TR), 2,300 ms; echo time (TE), $2.96 \mathrm{~ms}$; inversion time (TI) $900 \mathrm{~ms}$; flip angle (FA) $9^{\circ}$; field of view (FOV) $176 \times 256 \times 256 \mathrm{~mm}$; spatial resolution $1.0 \times 1.0 \times 1.0 \mathrm{~mm}^{3}$ for anatomical imaging, and segmentation and 3D flow-compensated multi-echo gradient-echo (GRE) images in axial plane $(\mathrm{TR}=33 \mathrm{~ms}$; first $\mathrm{TE}=4.5 \mathrm{~ms}$, uniform echo spacing $=5 \mathrm{~ms}$, last $\mathrm{TE}=29.5 \mathrm{~ms}$, number of echoes $=6 ; \mathrm{FA}=18^{\circ} ; \mathrm{FOV}=195 \times 240 \times 164 \mathrm{~mm}$; spatial resolution $=0.94 \times 0.94 \times 0.94 \mathrm{~mm}^{3}$ ) for QSM.

\section{QSM processing}

QSM was processed by a multi-scale dipole inversion algorithm implemented in QSMbox software package (https://gitlab.com/acostaj/QSMbox) (7,30). T1WI (i.e., MPRAGE) images were transferred into the QSM space by rigid-body registering to the first echo magnitude image from the GRE pulse sequence, using the SPM12 (Statistical Parametric Mapping) tool, (www. fil.ion.ucl.ac.uk/spm/software/spm12) (31) and skull stripped by multiplying with an SPM-based brain binary mask calculated by QSMbox. Co-registered skullstripped QSM and T1WI entered an automated multiatlas segmentation pipeline using dual, i.e., QSM/T1, contrast for delineation of DGM nuclei (32) implemented at a cloud-based platform (www.mricloud.org) (33). Subsequently, segmented volumes were eroded by one voxel, and mean bulk susceptibility values of the following regions of interest (ROI) were extracted: internal globus pallidus, external globus pallidus, putamen, caudate nucleus, thalamus, pulvinar, substantia nigra, red nucleus, dentate nucleus, subthalamic nucleus, and total white matter (Figure 1). Magnetic susceptibility values from both hemispheres were averaged and further analyzed as (I) raw uncorrected values and (II) values referenced to the mean bulk magnetic susceptibility of the total white matter. Notably, the total white matter was segmented by the automatic algorithm and its mean bulk susceptibility thus represents a highly reproducible operator independent measurement.

\section{T2* maps processing}

$\mathrm{T}^{*}$ relaxation maps were calculated from multi-echo GRE magnitude images using non-linear least squares fitting according to the Levenberg-Marquardt algorithm (MRI Processor v.1.1.6, ImageJ $1.51 \mathrm{k}$; T2* values were capped at $100 \mathrm{~ms}$, the maximum number of iterations was 100 , and forced no bias). $\mathrm{T} 2 *$ relaxation maps were used for wholebrain analysis.

\section{Whole-brain analysis}

The analysis was based on a procedure described previously (28). Briefly, a linear pre-registration (antsRegistration) and non-linear transformation (antsApplyTransformation) of susceptibility and T2* relaxation maps to the standard MNI template (MNI152 "ICBM 2009c Nonlinear Symmetric") was performed using ANTS package ver. 2.1.0 (34). To compensate for a small mismatch in registration, we applied a median filter followed by a maximum intensity filter, both calculated across a sphere with a diameter of $3 \mathrm{~mm}$. This approach was chosen empirically-we have visually compared the output images after eleven different combinations of filters (median spherical, median cubic, Gaussian spherical) with different kernel sizes applied. The selected combination of filters yielded images with a reasonable level of noise and detail and it appeared to be the most suitable approach for co-registration of susceptibility and $\mathrm{T} 2 *$ relaxation maps; the median filter removed both positive and negative signal values caused by vessels while the maximum intensity spherical filter (3 voxels in diameter) compensated for imperfections in co-registration of narrow structures (especially the gyri) to the MNI152 template.

In a post-hoc analysis, a mask of cortical voxels with a significant association of magnetic susceptibility and age was created. This mask was overlaid onto an anatomical T1 template and nine ROIs consisting of voxels from this mask were manually segmented using ITK-SNAP (www.itksnap. org) (35): precentral gyrus, postcentral gyrus, dorsolateral prefrontal cortex, medial prefrontal cortex, insular cortex, 

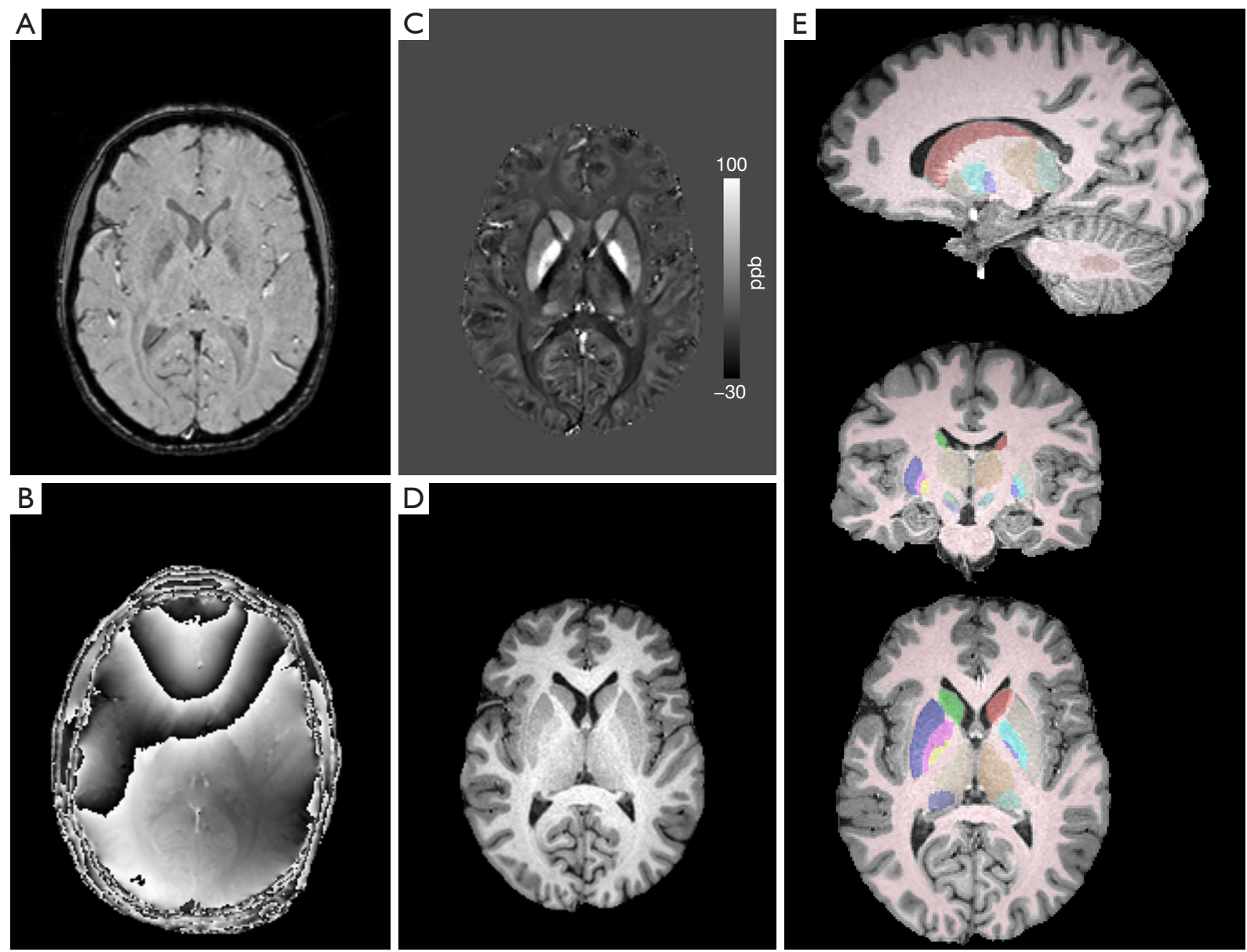

Figure 1 Illustration of MRI data processing. Example (A) magnitude and (B) raw phase (TE =24.5 ms) GRE images; (C) reconstructed QSM image and (D) corregistered skull-stripped T1-weighted anatomical image that were used for automatic multiatlas segmentation; (E) automatically segmented regions of interest overlaid onto anatomical image. ppb, parts per billion; GRE, gradient recalled echo; TE, echo time; QSM, quantitative susceptibility mapping.

precuneus, occipital cortex, occipito-temporal cortex, and cerebellar vermis (Figure S1). Individual mean bulk magnetic susceptibility values from these ROIs were retrieved and further analyzed as described for deep grey matter ROIs (25).

\section{Statistics}

Univariate regression analysis for regional age-related magnetic susceptibility changes was performed with linear and non-linear (quadratic and exponential) models and the model with the best fit based on Akaike information criterion with correction for small sample sizes (AICc), $\mathrm{R}^{2}$, and root mean squared error (RMSE) was selected. For linear relationships, regression slope $(\beta)$ was expressed in parts per billion (ppb)/year and its difference from zero was tested by the $\mathrm{F}$ test.

The exponential model was based on one-phase association and the susceptibility values were fitted with the following equation: susceptibility $=\mathrm{A}^{*}\left(1-\exp \left[-\mathrm{B}^{*}\right.\right.$ age $\left.]\right)$ $+\mathrm{C}$, where $\mathrm{A}, \mathrm{B}$, and $\mathrm{C}$ are tissue-specific parameters (25). Holm-Bonferroni method was applied to correct for multiple hypothesis testing. Statistical analyses were carried out using Prism 8 (GraphPad Software, San Diego, CA, USA).

Whole-brain analyses of age-related magnetic susceptibility and $\mathrm{T} 2$ * relaxation time changes were performed using SPM12. Statistical maps were generated using a cluster definition threshold of $\mathrm{P}<0.005$ (uncorrected) and clusterwise family-wise error (FWE)-corrected threshold of $\mathrm{P}<0.05$. Voxel-wise maps of linear regression 
slopes $\beta$ were visualized using MRIcroGL (http://www. nitrc.org/projects/mricrogl); anatomical locations of significant clusters were retrieved from the Atlas of Intrinsic Connectivity of Homotopic Areas (AICHA) (36).

\section{Results}

\section{ROI-based analysis}

The age-related increase of magnetic susceptibility in the basal ganglia followed a linear pattern, but in the thalamus and pulvinar, it followed a quadratic and exponential pattern, respectively (Figure 2, Table S1). Deep grey matter structures with a significant linear increase of raw bulk magnetic susceptibility included the red nucleus $(\beta=1.30$, $\mathrm{P}<0.001)$, putamen $(\beta=0.89, \mathrm{P}<0.001)$, substantia nigra $(\beta=0.76, P<0.001)$, dentate nucleus $(\beta=0.73, P=0.002)$, external globus pallidus $(\beta=0.62, \mathrm{P}<0.001)$, caudate nucleus $(\beta=0.46, P<0.001)$, and subthalamic nucleus $(\beta=0.43$, $\mathrm{P}=0.024)$. The effect of age was not significant for the internal globus pallidus $(\beta=0.20, \mathrm{P}=0.15)$. In the thalamus, magnetic susceptibility increased until the age of 40 years and decreased thereafter. In the pulvinar, susceptibility increase was followed by a relative flattening of the curve from the age of 40 years onwards. Analysis of magnetic susceptibility referenced to the total white matter value returned similar results (Figures S2,S3), whereby mean magnetic susceptibility in the total white matter showed a mild linear positive effect of age ( $\beta=0.03, \mathrm{P}=0.002)$.

\section{Whole-brain analyses}

Results of the whole-brain QSM analysis documenting magnetic susceptibility increase in ppb per year are shown in Figure 3 and Table 1. In addition to deep grey matter structures, multiple cortical regions with a positive association of magnetic susceptibility and age were identified in the precentral and postcentral areas, medial and dorsolateral prefrontal cortex, superior temporal gyrus and insula, posteriorly in the superior, lateral, and medial occipital gyri, precuneus, cuneus, posterior cingulate, fusiform, calcarine and lingual gyrus $(\mathrm{P}<0.05$, FWEcorrected). In the cerebellum, the vermis and tonsils showed a significant positive correlation with age $(\mathrm{P}<0.05$, FWEcorrected). Spatial coordinates and anatomical labeling of clusters with significant age-related magnetic susceptibility increase are listed in Table 1.

Several regions showed magnetic susceptibility decrease with age, although the effect was much smaller compared to areas with susceptibility increase reaching $\beta$ not greater than -0.2 ppb per year $(\mathrm{P}<0.05$, FWE-corrected $)$. These regions include distinct white matter areas, particularly the rostrum of the corpus callosum, external capsulae, mesencephalic tectum and tegmentum, and frontal and temporal subcortical regions. Several extracerebral regions, the choroid plexus, falx cerebri, and venous sinuses showed age-related susceptibility decrease with $\beta$ up to $-1.0 \mathrm{ppb}$ per year $(\mathrm{P}<0.05$, FWE-corrected).

Post-hoc analysis of magnetic susceptibility in cortical regions with a significant effect of age showed its linear increase in the postcentral, dorsolateral prefrontal, medial prefrontal, insular, occipital cortices, precuneus, and cerebellar vermis $(\beta \approx 0.2, \mathrm{P}<0.001)$. In the precentral gyrus and temporo-occipital gyrus, magnetic susceptibility followed a quadratic pattern with a relatively steep increase until the age of 40 years and flattening of the curve afterwards (Figure 4).

Results of $\mathrm{T} 2$ * analysis are shown in Figure $\mathrm{S} 4$ and Table S2. The results are similar to QSM however, a larger number of areas correlate with age. Compared to QSM, associations are evident also in the white matter.

\section{Discussion}

In this study, we quantified age-related changes of magnetic susceptibility in deep grey matter structures and the cerebral cortex in a cohort of adult healthy individuals using whole-brain and ROI-based analyses. We found a linear association between age and magnetic susceptibility in deep grey matter structures including the red nucleus, putamen, substantia nigra, dentate nucleus, external globus pallidus, and caudate nucleus. In the thalamus, quadratic association with age was observed with magnetic susceptibility increasing before and decreasing after the age of 40 years. Whole-brain QSM analysis documented that beyond these regions, there is a significant positive association between age and magnetic susceptibility in extensive areas of the cerebral cortex-in the frontal lobes, Rolandic area, insular area, precuneus, cuneus, posterior cingulum, and lingual gyrus. Whole-brain $\mathrm{T}^{*}$ analysis showed a similar pattern of age-related changes. In opposite to susceptibility, which showed a positive effect of age only in grey matter, areas of age-related $\mathrm{T} 2{ }^{*}$ relaxation times shortening overlapped grey and white matter structures. This is likely caused by the fact that in QSM, the paramagnetic effect of accumulating iron and the diamagnetic effect of maturating myelin counteract 


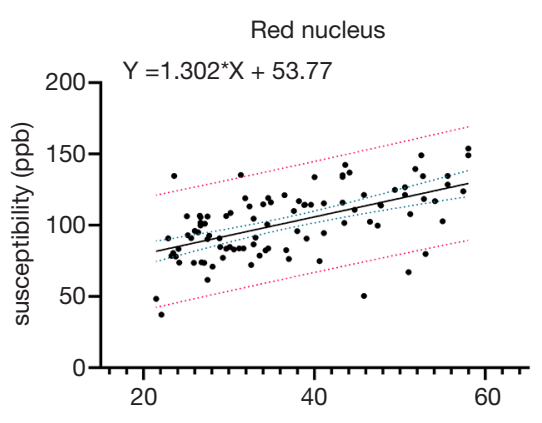

Dentate nucleus

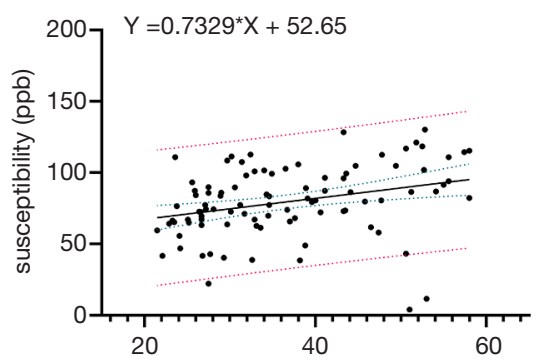

Subthalamic nucleus
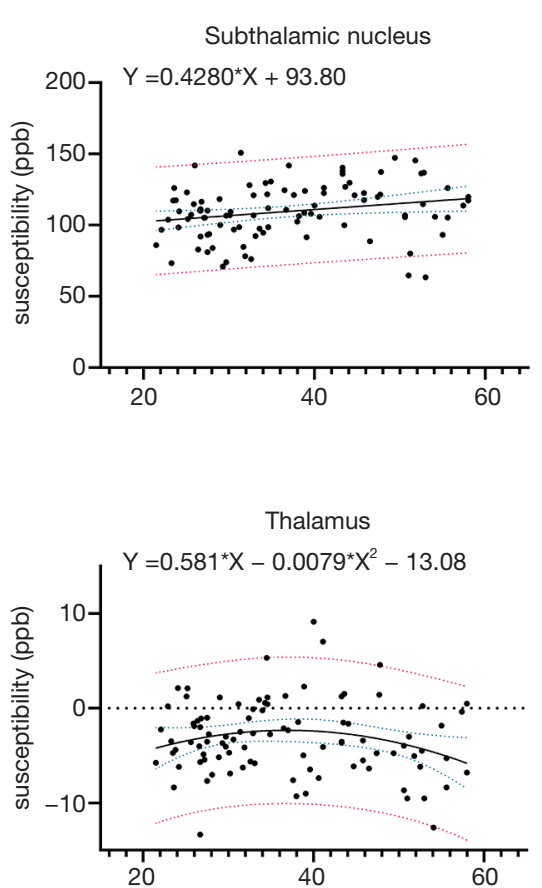
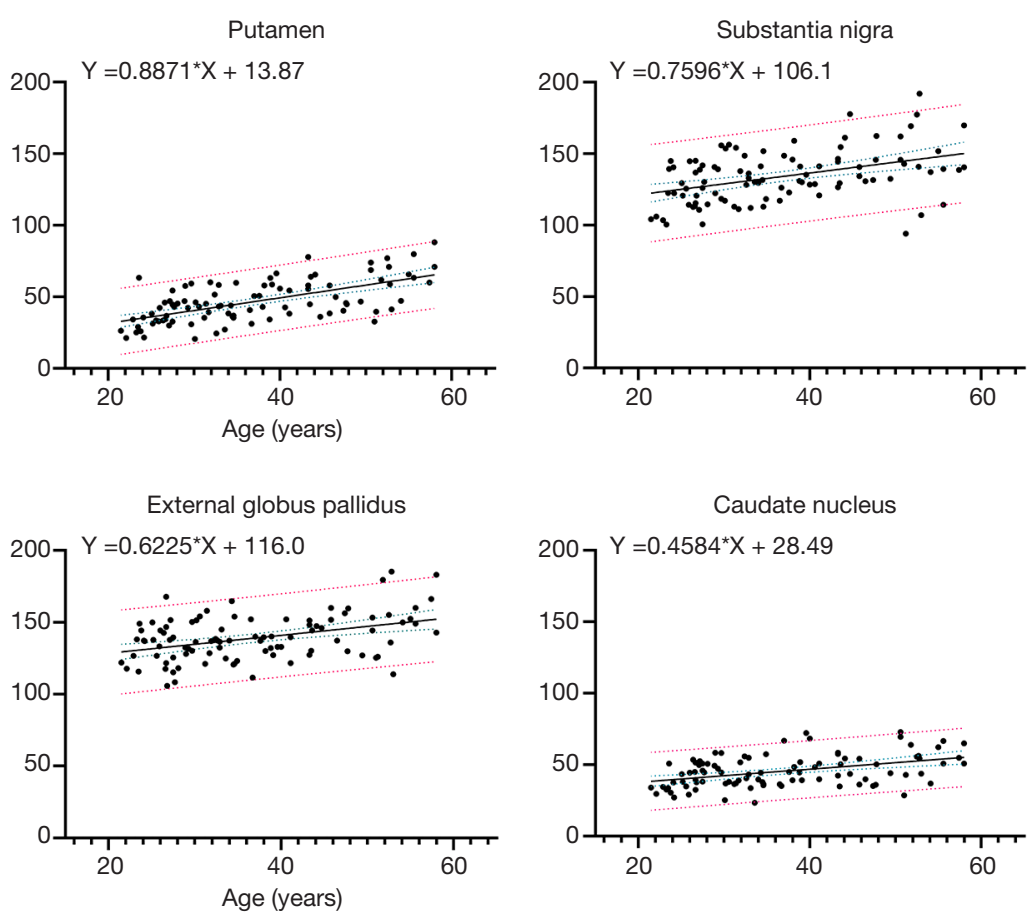

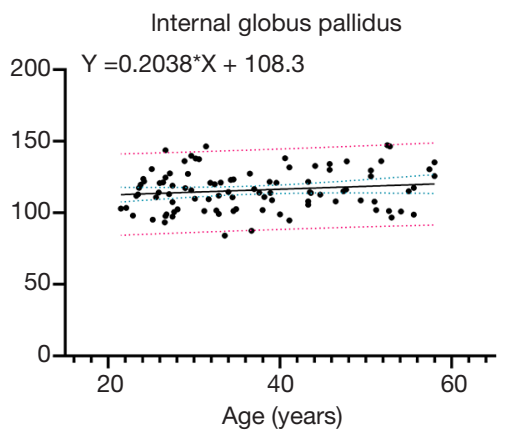

\begin{tabular}{l|c|c} 
& $P$ value & $R^{2}$ \\
\hline Red Nucleus & $<0.001^{*}$ & 0.33 \\
\hline Putamen & $<0.001^{*}$ & 0.39 \\
\hline Substantia Nigra & $<0.001^{*}$ & 0.18 \\
\hline Dentate & $0.002^{*}$ & 0.10 \\
\hline GP external & $<0.001^{\star}$ & 0.17 \\
\hline Caudate & $<0.001^{*}$ & 0.18 \\
\hline STN & $0.024^{*}$ & 0.05 \\
\hline GP internal & 0.15 & 0.02 \\
\hline Pulvinar & n.a. & 0.15 \\
\hline Thalamus & n.a. & 0.05 \\
\hline
\end{tabular}

Figure 2 Bulk magnetic susceptibility values in ppb in deep gray matter structures as a function of age with their respective regression fits and coefficients. Blue dotted lines represent $95 \%$ confidence bands of the regression fit and red dotted lines represent $95 \%$ population prediction bands. Please note different Y-axis range for thalamus and pulvinar. ppb, parts per billion; GP, globus pallidus, STN, subthalamic nucleus. 


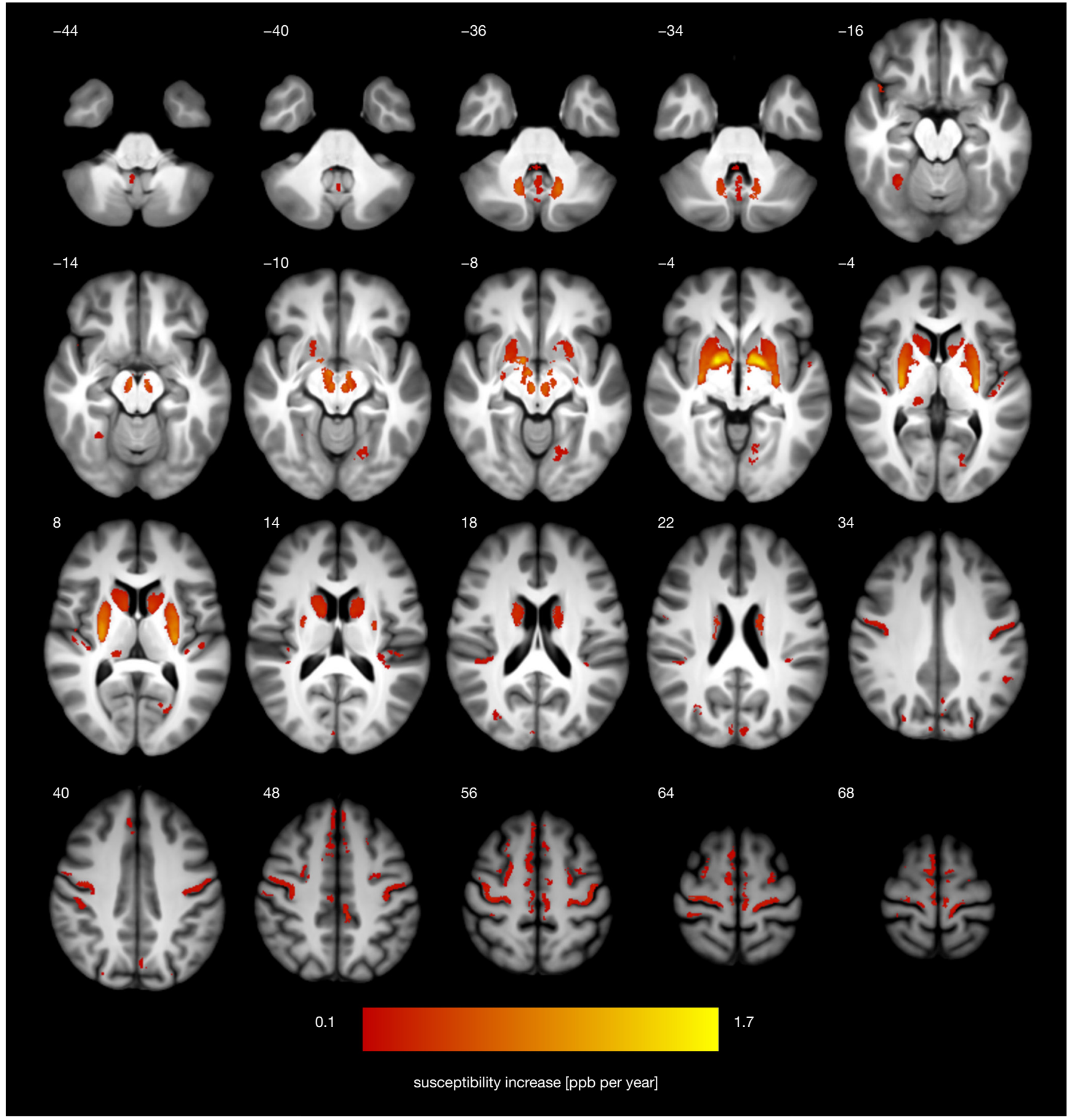

Figure 3 Statistical map of age-related susceptibility changes in the brain of normal adults represented as mean bulk susceptibility increase in ppb per year (thresholded at $\mathrm{P}<0.05$ at cluster level and corrected for family-wise error; initial cluster definition threshold was set to $\mathrm{P}<0.005$ uncorrected). Z-coordinate in the MNI space in [mm] is indicated for each slice. ppb, parts per billion; MNI, Montreal Neurological Institute. 
Table 1 Clusters with significant age-related susceptibility increase identified by whole brain analysis with corresponding anatomical locations

\begin{tabular}{|c|c|c|c|c|c|c|c|}
\hline \multirow{2}{*}{$\begin{array}{l}\text { Cluster volume } \\
{\left[\mathrm{mm}^{3}\right]}\end{array}$} & \multirow{2}{*}{$\begin{array}{l}\text { Peak susceptibility } \\
\text { change [ppb/year] }\end{array}$} & \multirow{2}{*}{$\begin{array}{l}\text { Peak T } \\
\text { value }\end{array}$} & \multicolumn{3}{|c|}{ MNI coordinates [mm] } & \multirow{2}{*}{ Peak structure } & \multirow{2}{*}{ Anatomical structures within cluster } \\
\hline & & & $x$ & Y & Z & & \\
\hline 10,477 & 1.7 & 10.7 & 16 & 1 & -5 & Putamen* & $\begin{array}{l}\text { Right putamen, pallidum, caudate, } \\
\text { substantia nigra, red nucelus* }\end{array}$ \\
\hline 10,018 & 1.5 & 10.3 & -14 & 1 & -4 & Putamen ${ }^{*}$ & $\begin{array}{l}\text { Left putamen, pallidum, caudate, sub- } \\
\text { stantia nigra, red nucleus }{ }^{\star}\end{array}$ \\
\hline 369 & 0.7 & 4.9 & 15 & -58 & -37 & Dentate* $^{\star}$ & Right dentate* $^{*}$ \\
\hline 3,126 & 0.5 & 7.0 & -41 & -11 & 56 & Precentral gyrus & $\begin{array}{l}\text { Left precentral gyrus, paracentral lob- } \\
\text { ule }\end{array}$ \\
\hline 685 & 0.5 & 4.9 & -23 & -68 & 7 & Calcarine gyrus & $\begin{array}{l}\text { Left calcarine, lingual, fusiform gyri, } \\
\text { parietooccipital sulcus }\end{array}$ \\
\hline 3,628 & 0.4 & 6.4 & 23 & -22 & 63 & Precentral gyrus & $\begin{array}{l}\text { Right precentral gyrus, paracentral } \\
\text { lobule }\end{array}$ \\
\hline 811 & 0.4 & 4.8 & 45 & -16 & 0 & $\begin{array}{l}\text { Superior temporal } \\
\text { gyrus }\end{array}$ & $\begin{array}{l}\text { Right superior temporal, supramargin- } \\
\text { al gyri, posterior insula }\end{array}$ \\
\hline 1,902 & 0.4 & 5.0 & -8 & -35 & 47 & Cingulate sulcus & $\begin{array}{l}\text { Left cingulate sulcus, superior frontal } \\
\text { gyrus, paracentral lobule }\end{array}$ \\
\hline 252 & 0.3 & 6.4 & 29 & -54 & -16 & Fusiform gyrus & Right fusiform, lateral occipital gyri \\
\hline 303 & 0.3 & 4.7 & 5 & -88 & 30 & Superior occipital gyrus & $\begin{array}{l}\text { Right superior occipital, calcarine gyri, } \\
\text { cuneus }\end{array}$ \\
\hline 1,503 & 0.3 & 5.4 & 23 & 11 & 63 & Superior frontal sulcus & $\begin{array}{l}\text { Right superior frontal gyrus and sul- } \\
\text { cus, precentral sulcus }\end{array}$ \\
\hline 193 & 0.3 & 4.9 & -4 & -81 & 26 & Cuneus & $\begin{array}{l}\text { Left cuneus, calcarine, superior occip- } \\
\text { ital gyri }\end{array}$ \\
\hline 383 & 0.3 & 4.1 & 35 & 11 & 25 & Inferior frontal gyrus & $\begin{array}{l}\text { Right inferior frontal gyrus, precentral } \\
\text { sulcus }\end{array}$ \\
\hline 326 & 0.3 & 4.9 & -2 & -74 & 34 & Parietooccipital sulcus & Left parietooccipital sulcus, precuneus \\
\hline 635 & 0.2 & 4.6 & 45 & -25 & 43 & Postcentral sulcus & Right postcentral sulcus and gyrus \\
\hline 1,241 & 0.2 & 4.7 & -33 & -4 & 54 & Superior frontal gyrus & $\begin{array}{l}\text { Left superior frontal sulcus and gyrus, } \\
\text { precentral sulcus }\end{array}$ \\
\hline 427 & 0.2 & 4.2 & 26 & -73 & 29 & Middle occipital gyrus & $\begin{array}{l}\text { Right middle occipital, angular gyri, } \\
\text { intraoccipital, intraparietal sulci }\end{array}$ \\
\hline 239 & 0.2 & 3.9 & -50 & -47 & 32 & Supramarginal gyrus & Left supramarginal, inferior parietal gyri \\
\hline 373 & 0.2 & 4.1 & -6 & -66 & -58 & Cerebellum & Left and right cerebellar tonsils \\
\hline
\end{tabular}

${ }^{\star}$ Deep gray matter structures. 


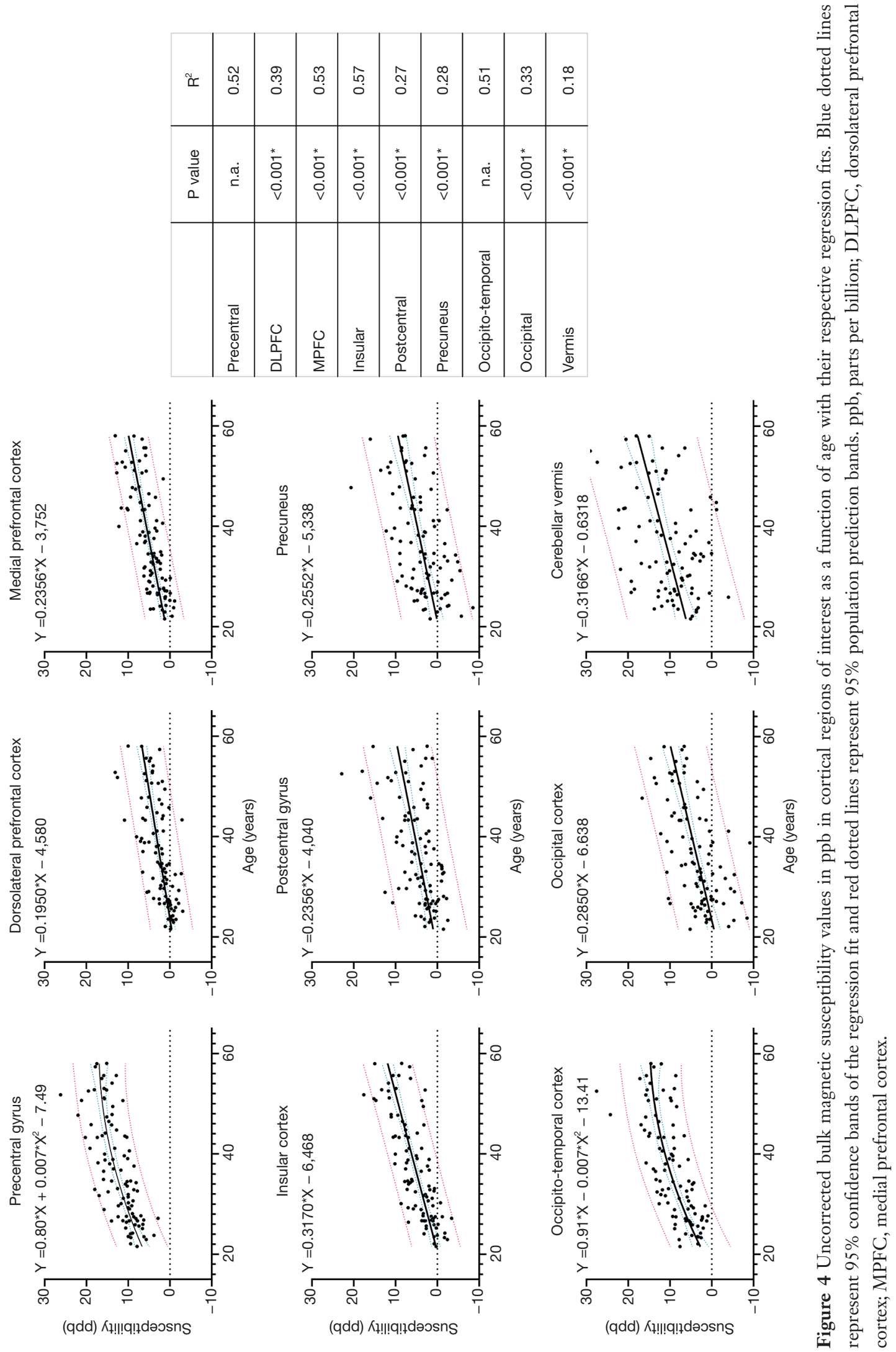


each other in the white matter, while in $\mathrm{T} 2{ }^{*}$ maps, both, iron accumulation and myelin maturation with aging, cause local magnetic field inhomogeneities and thus contribute to the shortening of $\mathrm{T} 2 *$ relaxation times. This effect was particularly noted in subcortical white matter regions which are known to accumulate iron. Comparison of QSM and $\mathrm{T}^{*}$ map analyses indicates that the former may be more suitable for the assessment of age-related iron changes in the cortical regions.

It is well known that the trajectory of brain iron accumulation during the lifespan is not linear. A sharp increase of tissue iron concentration (15) and subsequently of magnetic susceptibility $(25,27,37)$ during the first two decades is followed by flattening of the curve or even decrease at old age. Results of this study indicate the evolution of susceptibility values is linear between the age of 20 and 60 years for most deep grey matter and cortical structures. It is likely that regions that showed an exponential relationship between age and magnetic susceptibility in the current study, i.e., pulvinar, motor and occipito-temporal cortices, exhibit less abrupt initial iron accumulation with flattening of the curve shifted to an older age.

We found the largest effect of age on magnetic susceptibility (the steepest regression slope) in the red nucleus and putamen, which is consistent with previous studies $(11,27,38)$. The red nucleus has been previously less investigated and, in some studies, the absolute magnitude of susceptibility increase was higher for the red nucleus than for the putamen $(22,27,29)$, similarly as in our study. Our observation of milder gradual increase of magnetic susceptibility in the caudate nucleus, substantia nigra, and dentate nucleus is also concordant with postmortem evidence (16) and several previous in vivo MRI studies $(9,13,14,27,28)$.

In the pallidum, previous works reported contradictory findings including no age-related susceptibility changes $(23,27,28)$, or mild iron accumulation during adulthood (22). A meta-analysis of 20 MRI studies that estimated iron content in the caudate nucleus, globus pallidus, putamen, red nucleus, and substantia nigra, documented the lowest age-related differences in globus pallidus although it was generally rich in iron in absolute value (11). Studies that included infants showed an exponential pattern with a steeply increasing iron concentration as well as magnetic susceptibility from birth and a plateau from the $3^{\text {rd }}$ decade onwards $(13,14,16,38-40)$. However, these studies did not aim at analyzing specifically the pallidal iron content trajectory during the adult lifespan. Our results indicate that the reason for contradictory findings may be the differential contribution of external and internal globus pallidus depending on segmentation strategy. In contrast to the internal part, the external part, which is adjacent to the putamen, showed a linear increase of susceptibility during aging in the current study. A similar effect was observed for $\mathrm{R} 2$ * transverse relaxivity in another study that segmented internal and external globus pallidus (29). These results indicate distinct regulation of iron concentration in pallidal subregions and suggest that internal and external globus pallidus should be analyzed separately for proper assessment of disease-related changes in this structure.

Thalamus presents an overall low iron content and it was suggested that myelin contributes by a large degree to its susceptibility masking the effect of small age-related changes in iron content (41). On the other hand, the bidirectional pattern with an initial increase until the age of 40 years followed later by a decrease of magnetic susceptibility observed in the current and a previous study (42) conspicuously matches age-related changes in the thalamic iron concentration measured in post mortem tissue (15). It is thus likely that thalamic susceptibility is mostly driven by the effect of iron, similarly as in the basal ganglia. Contrary to some previous studies (27), we and others have found a positive age-related magnetic susceptibility increase in the pulvinar $(42,43)$. It is interesting to point up the differences between pulvinar and other thalamic nuclei. The pulvinar, although being a posterior part of the thalamus, is visually discernible on QSM and behaves unlike other thalamic nuclei. Zhang et al. (42) suggests that in the medial and lateral nuclei of the thalamus susceptibility follows a bidirectional pattern while anterior nuclei and pulvinar display gradual susceptibility increase with age. Overall, there is converging evidence that MRI metrics sensitive to iron content should be analyzed separately for pulvinar and other thalamic nuclei.

Age-related changes in iron distribution in the cerebral and cerebellar cortex in vivo have been much less investigated. It may be related to the fact that the cerebral cortex has more complex and variable anatomy than deep grey matter nuclei and its automatic analysis at a group level requires non-linear transformation to match corresponding regions among subjects. Further challenges include the thinning of the cortex and widening of subarachnoid cerebrospinal fluid spaces in cerebral sulci with cerebral atrophy that accompanies aging. Results of the current whole-brain analysis largely corroborate findings from a single previous voxel-based 
QSM study in that cortical magnetic susceptibility evolves unevenly during aging $(28,29)$. The strongest effect of age was found in areas involved in the motor (Rolandic area, premotor cortex, cerebellar vermis), cognitive (dorsolateral prefrontal cortex, insular region, precuneus), and visual (cuneus, posterior cingulum, lingual and fusiform gyri) cortical areas. This is also in line with the direct measurement of iron concentration in post mortem tissue (15) which indicates that iron has an important contribution to magnetic susceptibility in the cortex. Overall, structures that accumulate iron with aging contain dopaminergic iron-dependent pathways and it was hypothesized that both, changes in iron content and dopaminergic transmission may be responsible for behavioral changes during aging $(27,28,44)$.

In the white matter, magnetic susceptibility is predominantly affected by diamagnetic myelin and it was previously shown to follow a trajectory of initial susceptibility decrease reaching its minimum between 25 and 45 years of age, followed by an increase, consistent with myelin maturation and decay in the course of normal brain development (25). We found a small but significant positive effect of age on the magnetic susceptibility of the total white matter, similar to a previous study (30) suggesting that susceptibility in most white matter regions was beyond its minimum in the examined age group. However, the whole-brain QSM analysis revealed white matter regions with a small negative effect of age corresponding to areas with an age-related decrease of $\mathrm{T}^{*}$ relaxation time, predominantly in the fronto-temporal subcortical areas and rostral corpus callosum which are regions where myelin maturates in late adulthood (45).

In line with previous studies $(25,28)$ which demonstrated that the effect of age on magnetic susceptibility outweighs the impact of reference adjustment, we have used raw unreferenced values for the main analysis in order to avoid bias introduced by manual reference region placement. Secondary analysis with magnetic susceptibility referenced to the mean value of total white matter confirmed a negligible effect of susceptibility adjustment.

This study has several limitations. Firstly, its crosssectional nature does not allow direct analysis of individual regional magnetic susceptibility values evolution throughout aging. A longitudinal study will be necessary to confirm the relationship between aging and magnetic susceptibility. Secondly, the age spectrum of participants spanning 20 60 years does not include adolescents and seniors; the results are thus not generalizable to these populations. The primary aim of this study was to provide reference values for multiple sclerosis patients, but the results may be as well used for other diseases with an adult onset, e.g., Huntington disease, Wilson disease, or neuromyelitis optica $(46,47)$. Thirdly, magnetic susceptibility is only a surrogate marker of iron concentration and is also influenced by other substances such as diamagnetic myelin. However, an excellent match of susceptibility values with previously published directly measured iron concentrations indicates that for grey matter structures, magnetic susceptibility is dominantly influenced by iron and the confounding effect of myelin is rather small. Fourthly, voxel-based analysis of the brain involves non-rigid warping of anatomy to match the template. This is inherently associated with registration errors that may lead to false-negative agerelated associations for some brain areas. Lastly, susceptibility changes are presumably influenced not only by absolute iron content but also by changes in the relative iron concentration secondary to age-related reductions in volumes of evaluated structures (48). However, such complex relationships cannot be inferred from this cross-sectional study.

In conclusion, we have demonstrated that iron accumulates in specific brain regions with different patterns. Regions most prone to accumulate iron are the ones involved in the motor, visual, and cognitive functions. Iron accumulation follows a linear pattern during adult lifespan in the basal ganglia and most cortical areas but in the thalamus, pulvinar, precentral and occipito-temporal cortices, it follows a quadratic or exponential pattern. Changes in iron content during aging are different in the pulvinar and the rest of the thalamus. We suggest that whole brain analysis of magnetic susceptibility and segmentation of thalamic and pallidal subregions is beneficial for proper assessment of aging and disease-related changes in cerebral iron content. Departure from normal patterns of regional brain iron trajectories during aging might be helpful in the detection of subtle neurodegenerative and neuroinflammatory processes.

\section{Acknowledgments}

This work was presented as a poster at ECTRIMS virtual congress 2020.

Funding: This study was supported by the Czech Ministry of Health grant (NV18-08-00062, and RVO VFN64165), by the Charles University in Prague grant (PROGRES Q27) and by Roche company.

\section{Footnote}

Conflicts of Interest: All authors have completed the ICMJE 
uniform disclosure form (available at http://dx.doi. org/10.21037/qims-21-87). Dr. PD reports funding from Czech Ministry of Health, grant No. NU21-0400535 and European Union's Horizon 2020 research and innovation programme, grant No. 633190, and advisory board payment from Alexion Pharmaceuticals. Dr. MV reports funding from Czech Ministry of Health, grant No. NV 18-04-00168, honoraria for lectures and presentation from Biogen Idec, Novartis, Sanofi Genzyme, Merck Serono and Teva and support for attending meetings and/ or travel from Biogen Idec, Novartis, Sanofi Genzyme. Dr. DH reports honoraria for lectures and presentation from Biogen Idec, Novartis, Sanofi, Roche and Merck and support for attending meetings and/or travel from Biogen Idec, Novartis, Sanofi, Roche and Merck. Dr. JK reports funding from Czech Ministry of Health, grant No. NV 1804-00168 and support for attending meetings and/or travel Biogen Idec, Novartis, Sanofi Genzyme. Dr. LL serves as an unpaid editorial board member of Quantitative Imaging in Medicine and Surgery. The other authors have no conflicts of interest to declare.

Ethical Statement: The authors are accountable for all aspects of the work in ensuring that questions related to the accuracy or integrity of any part of the work are appropriately investigated and resolved. The study was conducted in accordance with the Declaration of Helsinki (as revised in 2013). This study was approved by the Ethics Committee of the General University Hospital in Prague (ID1018/17), and all subjects signed informed consent.

Open Access Statement: This is an Open Access article distributed in accordance with the Creative Commons Attribution-NonCommercial-NoDerivs 4.0 International License (CC BY-NC-ND 4.0), which permits the noncommercial replication and distribution of the article with the strict proviso that no changes or edits are made and the original work is properly cited (including links to both the formal publication through the relevant DOI and the license). See: https://creativecommons.org/licenses/by-nc-nd/4.0/.

\section{References}

1. Callaghan MF, Freund P, Draganski B, Anderson E, Cappelletti M, Chowdhury R, Diedrichsen J, FitzGerald THB, Smittenaar P, Helms G, Lutti A, Weiskopf N. Widespread age-related differences in the human brain microstructure revealed by quantitative magnetic resonance imaging. Neurobiol Aging 2014;35:1862-72.

2. Lorio S, Lutti A, Kherif F, Ruef A, Dukart J, Chowdhury R, Frackowiak RS, Ashburner J, Helms G, Weiskopf $\mathrm{N}$, Draganski B. Disentangling in vivo the effects of iron content and atrophy on the ageing human brain. NeuroImage 2014;103:280-9.

3. Pfefferbaum A, Adalsteinsson E, Rohlfing T, Sullivan EV. MRI estimates of brain iron concentration in normal aging: Comparison of field-dependent (FDRI) and phase (SWI) methods. NeuroImage 2009;47:493-500.

4. Taubert M, Roggenhofer E, Melie-Garcia L, Muller S, Lehmann N, Preisig M, Vollenweider P, MarquesVidal P, Lutti A, Kherif F, Draganski B. Converging patterns of aging-associated brain volume loss and tissue microstructure differences. Neurobiol Aging 2020;88:108-18.

5. Wang Y, Liu T. Quantitative susceptibility mapping (QSM): Decoding MRI data for a tissue magnetic biomarker. Magn Reson Med 2015;73:82-101.

6. Langkammer C, Bredies K, Poser BA, Barth M, Reishofer G, Fan AP, Bilgic B, Fazekas F, Mainero C, Ropele S. Fast quantitative susceptibility mapping using $3 \mathrm{D}$ EPI and total generalized variation. NeuroImage 2015;111:622-30.

7. Acosta-Cabronero J, Milovic C, Mattern H, Tejos C, Speck O, Callaghan MF. A robust multi-scale approach to quantitative susceptibility mapping. NeuroImage 2018;183:7-24.

8. Dusek P, Dezortova M, Wuerfel J. Imaging of iron. Int Rev Neurobiol 2013;110:195-239.

9. Burgetova A, Seidl Z, Krasensky J, Horakova D, Vaneckova M. Multiple sclerosis and the accumulation of iron in the Basal Ganglia: quantitative assessment of brain iron using MRI t(2) relaxometry. Eur Neurol 2010;63:136-43.

10. Connor JR, Menzies SL, Martin SMS, Mufson EJ. Cellular distribution of transferrin, ferritin, and iron in normal and aged human brains. J Neurosci Res 1990;27:595-611.

11. Daugherty A, Raz N. Age-related differences in iron content of subcortical nuclei observed in vivo: a metaanalysis. NeuroImage 2013;70:113-21.

12. Schipper HM. Neurodegeneration with brain iron accumulation - clinical syndromes and neuroimaging. Biochim Biophys Acta 2012;1822:350-60.

13. Ward RJ, Zucca FA, Duyn JH, Crichton RR, Zecca L. The role of iron in brain ageing and neurodegenerative disorders. Lancet Neurol 2014;13:1045-60.

14. Ghadery C, Pirpamer L, Hofer E, Langkammer C, Petrovic K, Loitfelder M, Schwingenschuh P, Seiler S, 
Duering M, Jouvent E, Schmidt H, Fazekas F, Mangin J-F, Chabriat H, Dichgans M, Ropele S, Schmidt R. R2* mapping for brain iron: associations with cognition in normal aging. Neurobiol Aging 2015;36:925-32.

15. Hallgren B, Sourander P. The effect of age on the non-haemin iron in the human brain. J Neurochem 1958;3:41-51.

16. Ramos P, Santos A, Pinto NR, Mendes R, Magalhães T, Almeida A. Iron levels in the human brain: a post-mortem study of anatomical region differences and age-related changes. J Trace Elem Med Biol 2014;28:13-7.

17. Li J, Cao F, Yin H, Huang Z, Lin Z, Mao N, Sun B, Wang G. Ferroptosis: past, present and future. Cell Death Dis 2020;11:88.

18. Schipper HM. Brain iron deposition and the free radical-mitochondrial theory of ageing. Ageing Res Rev 2004;3:265-301.

19. Daugherty AM, Haacke EM, Raz N. Striatal Iron Content Predicts Its Shrinkage and Changes in Verbal Working Memory after Two Years in Healthy Adults. J Neurosci 2015;35:6731-43.

20. Kalpouzos G, Garzón B, Sitnikov R, Heiland C, Salami A, Persson J, Bäckman L. Higher Striatal Iron Concentration is Linked to Frontostriatal Underactivation and Poorer Memory in Normal Aging. Cereb Cortex 2017;27:3427-36.

21. Aquino D, Bizzi A, Grisoli M, Garavaglia B, Bruzzone MG, Nardocci N, Savoiardo M, Chiapparini L. Agerelated Iron Deposition in the Basal Ganglia: Quantitative Analysis in Healthy Subjects. Radiology 2009;252:165-72.

22. Bilgic B, Pfefferbaum A, Rohlfing T, Sullivan EV, Adalsteinsson E. MRI estimates of brain iron concentration in normal aging using quantitative susceptibility mapping. NeuroImage 2012;59:2625-35.

23. Gong NJ, Wong CS, Hui ES, Chan CC, Leung LM. Hemisphere, gender and age-related effects on iron deposition in deep gray matter revealed by quantitative susceptibility mapping. NMR Biomed 2015;28:1267-74.

24. Haacke EM, Miao Y, Liu M, Habib CA, Katkuri Y, Liu T, Yang Z, Lang Z, Hu J, Wu J. Correlation of putative iron content as represented by changes in R2* and phase with age in deep gray matter of healthy adults. J Magn Reson Imaging 2010;32:561-76.

25. Li W, Wu B, Batrachenko A, Bancroft-Wu V, Morey RA, Shashi V, Langkammer C, Bellis MDD, Ropele S, Song AW, Liu C. Differential developmental trajectories of magnetic susceptibility in human brain gray and white matter over the lifespan. Hum Brain Mapp
2014;35:2698-713.

26. Liu M, Liu S, Ghassaban K, Zheng W, Dicicco D, Miao Y, Habib C, Jazmati T, Haacke EM. Assessing global and regional iron content in deep gray matter as a function of age using susceptibility mapping. J Magn Reson Imaging 2016;44:59-71.

27. Persson N, Wu J, Zhang Q, Liu T, Shen J, Bao R, Ni M, Liu T, Wang Y, Spincemaille P. Age and Sex Related Differences in Subcortical Brain Iron Concentrations among Healthy Adults. NeuroImage 2015;122:385-98.

28. Acosta-Cabronero J, Betts MJ, Cardenas-Blanco A, Yang S, Nestor PJ. In Vivo MRI Mapping of Brain Iron Deposition across the Adult Lifespan. J Neurosci 2016;36:364-74.

29. Betts MJ, Acosta-Cabronero J, Cardenas-Blanco A, Nestor PJ, Düzel E. High-resolution characterisation of the aging brain using simultaneous quantitative susceptibility mapping (QSM) and R2* measurements at 7T. NeuroImage 2016;138:43-63.

30. Milovic C, Prieto C, Bilgic B, Uribe S, Acosta-Cabronero J, Irarrazaval P, Tejos C. Comparison of parameter optimization methods for quantitative susceptibility mapping. Magn Reson Med 2021;85:480-94.

31. Huhdanpaa H, Hwang DH, Gasparian GG, Booker MT, Cen Y, Lerner A, Boyko OB, Go JL, Kim PE, Rajamohan A, Law M, Shiroishi MS. Image Coregistration: Quantitative Processing Framework for the Assessment of Brain Lesions. J Digit Imaging 2014;27:369-79.

32. Li X, Chen L, Kutten K, Ceritoglu C, Li Y, Kang N, Hsu J, Qiao Y, Wei H, Liu C, Miller M, Mori S, Yousem DM, van Zijl P, Faria AV. Multi-Atlas Tool for Automated Segmentation of Brain Gray Matter Nuclei and Quantification of their Magnetic Susceptibility. NeuroImage 2019;191:337-49.

33. Mori S, Wu D, Ceritoglu C, Li Y, Kolasny A, Vaillant MA, Faria AV, Oishi K, Miller MI. MRICloud: Delivering High-Throughput MRI Neuroinformatics as Cloud-Based Software as a Service. Comput Sci Eng 2016;18:21-35.

34. Avants BB, Tustison NJ, Song G, Gee JC. Ants: Opensource tools for normalization and neuroanatomy. HeanetIe 2009;10:1-11.

35. Yushkevich PA, Piven J, Hazlett HC, Smith RG, Ho S, Gee JC, Gerig G. User-guided 3D active contour segmentation of anatomical structures: significantly improved efficiency and reliability. NeuroImage 2006;31:1116-28.

36. Joliot M, Jobard G, Naveau M, Delcroix N, Petit L, Zago L, Crivello F, Mellet E, Mazoyer B, Tzourio-Mazoyer N. AICHA: An atlas of intrinsic connectivity of homotopic areas. J Neurosci Methods 2015;254:46-59. 
37. van der Weijden MCM, van Laar PJ, Lambrechts RA, Verbeek DS, Tijssen MAJ. Cortical pencil lining on SWI MRI in NBIA and healthy aging. BMC Neurol 2019;19:233.

38. Liu C, Wei H, Gong NJ, Cronin M, Dibb R, Decker K. Quantitative Susceptibility Mapping: Contrast Mechanisms and Clinical Applications. Tomography 2015;1:3-17.

39. Ning N, Liu C, Wu P, Hu Y, Zhang W, Zhang L, Li M, Gho SM, Kim DH, Guo H, Yang J, Jin C. Spatiotemporal variations of magnetic susceptibility in the deep gray matter nuclei from 1 month to 6 years: A quantitative susceptibility mapping study. J Magn Reson Imaging 2019;49:1600-9.

40. Peterson ET, Kwon D, Luna B, Larsen B, Prouty D, Bellis MDD, Voyvodic J, Liu C, Li W, Pohl KM, Sullivan EV, Pfefferbaum A. Distribution of brain iron accrual in adolescence: Evidence from cross-sectional and longitudinal analysis. Hum Brain Mapp 2019;40:1480-95.

41. Hagemeier J, Dwyer MG, Bergsland N, Schweser F, Magnano CR, Heininen-Brown M, Ramasamy DP, Carl E, Kennedy C, Melia R, Polak P, Deistung A, Geurts JJG, Reichenbach JR, Zivadinov R. Effect of age on MRI phase behavior in the subcortical deep gray matter of healthy individuals. Am J Neuroradiol 2013;34:2144-51.

42. Zhang Y, Wei H, Cronin MJ, He N, Yan F, Liu C. Longitudinal atlas for normative human brain development and aging over the lifespan using quantitative susceptibility mapping. NeuroImage 2018;171:176-89.

43. Burgetova A, Dusek P, Vaneckova M, Horakova D, Langkammer C, Krasensky J, Sobisek L, Matras P, Masek M, Seidl Z. Thalamic Iron Differentiates PrimaryProgressive and Relapsing-Remitting Multiple Sclerosis. Am J Neuroradiol 2017;38:1079-86.

44. Steiger TK, Weiskopf N, Bunzeck N. Iron Level and Myelin Content in the Ventral Striatum Predict Memory Performance in the Aging Brain. J Neurosci 2016;36:3552-8.

45. Branson HM. Normal Myelination: A Practical Pictorial Review. Neuroimaging Clin N Am 2013;23:183-95.

46. Dezortova M, Lescinskij A, Dusek P, Herynek V, AcostaCabronero J, Bruha R, Jiru F, Robinson SD, Hajek M. Multiparametric Quantitative Brain MRI in Neurological and Hepatic Forms of Wilson's Disease. J Magn Reson Imaging 2020;51:1829-35.

47. Pudlac A, Burgetova A, Dusek P, Nytrova P, Vaneckova M, Horakova D, Krasensky J, Lambert L. Deep Gray Matter Iron Content in Neuromyelitis Optica and Multiple Sclerosis. Biomed Res Int 2020;2020:6492786.

48. Schweser F, Hagemeier J, Dwyer MG, Bergsland N, Hametner S, Weinstock-Guttman B, Zivadinov R. Decreasing brain iron in multiple sclerosis: The difference between concentration and content in iron MRI. Hum Brain Mapp 2021;42:1463-74.
Cite this article as: Burgetova R, Dusek P, Burgetova A, Pudlac A, Vaneckova M, Horakova D, Krasensky J, Varga Z, Lambert L. Age-related magnetic susceptibility changes in deep grey matter and cerebral cortex of normal young and middleaged adults depicted by whole brain analysis. Quant Imaging Med Surg 2021;11(9):3906-3919. doi: 10.21037/qims-21-87 

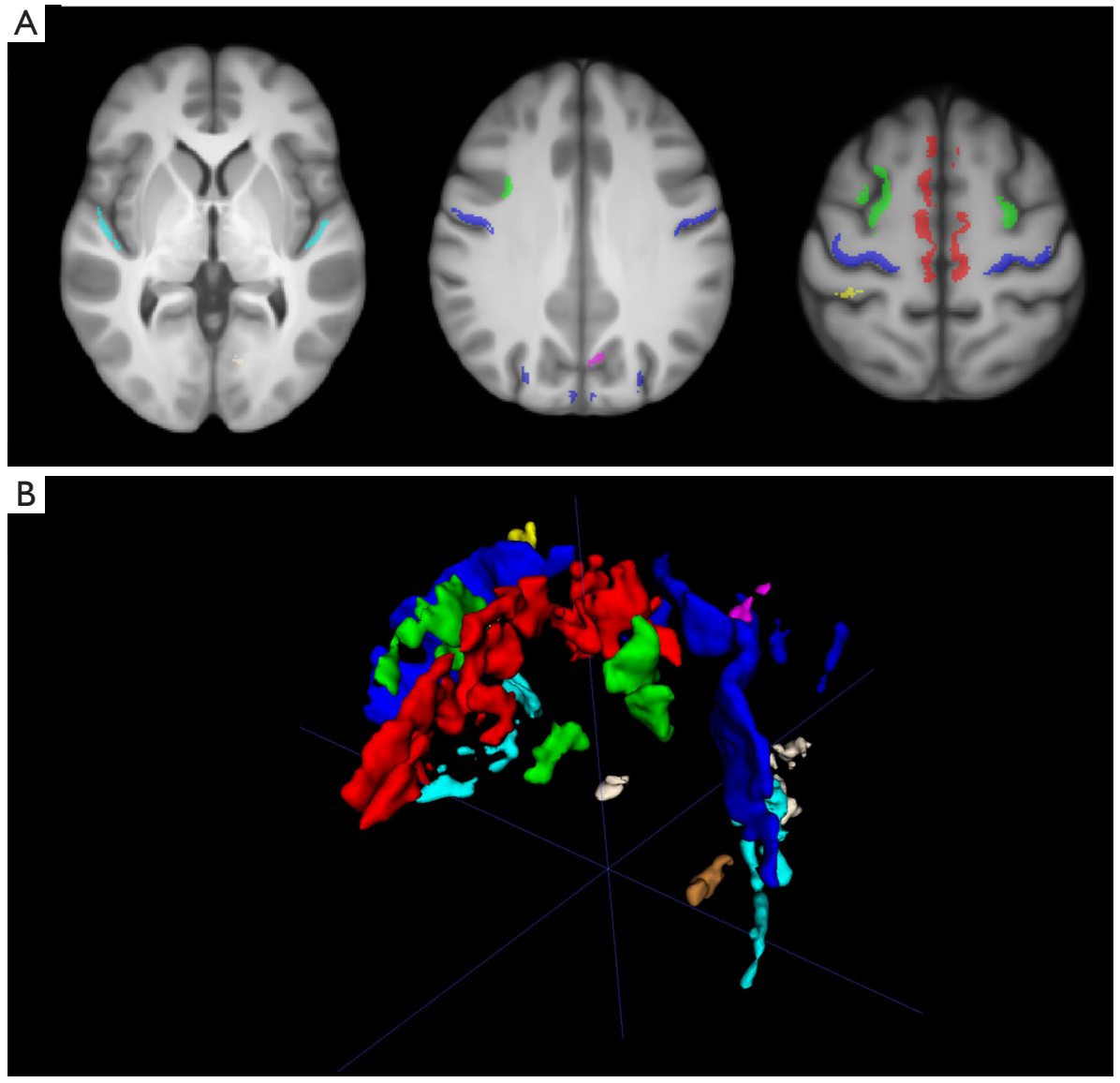

Figure S1 Cortical regions of interest manually segmented on a mask containing cortical voxels with positive association between magnetic susceptibility and age are shown A) on axial T1WI anatomical images and B) as an 3D reconstruction (left frontolateral view). Cortical ROIs include precentral gyrus (blue), postcentral gyrus (yellow), dorslolateral prefrontal cortex (green), medial prefrontal cortex (red), insular cortex (cyan), precuneus (magenta), occipital cortex (dark blue), occipito-temporal cortex (beige), and cerebellar vermis (brown). Abbreviations: T1WI = T1-weighted image; ROI = region of interest. 
Table S1 Comparison of linear, quadratic, and exponential models for age-related susceptibility changes (best model fit based on Akaike information criterion with correction for small sample sizes, $\mathrm{R}^{2}$, and RMSE is shown in blue)

\begin{tabular}{|c|c|c|c|c|c|c|c|c|c|c|c|c|}
\hline \multirow[b]{2}{*}{ Region } & \multicolumn{12}{|c|}{ Model } \\
\hline & $\mathrm{R}^{2}$ & $\mathrm{R}^{2}$ adj & RMSE & $\mathrm{AlCc}$ & $\mathrm{R}^{2}$ & $\mathrm{R}^{2}$ adj & RMSE & $\mathrm{AICc}$ & $\mathrm{R}^{2}$ & $\mathrm{R}^{2}$ adj & RMSE & $\mathrm{AlCc}$ \\
\hline red nucleus & 0.33 & 0.32 & 19.39 & 568.6 & 0.33 & 0.31 & 19.38 & 570.6 & 0.33 & 0.31 & 19.37 & 570.5 \\
\hline putamen & 0.39 & 0.39 & 11.38 & 467.3 & 0.39 & 0.38 & 11.38 & 469.5 & 0.39 & 0.38 & 11.38 & 469.5 \\
\hline dentate nucleus & 0.09 & 0.08 & 23.42 & 604.5 & 0.10 & 0.08 & 23.41 & 606.5 & 0.10 & 0.08 & 23.39 & 606.4 \\
\hline external globus pallidus & 0.17 & 0.16 & 14.4 & 512 & 0.19 & 0.17 & 14.24 & 512 & 0.17 & 0.15 & 14.4 & 514.2 \\
\hline caudate nucleus & 0.18 & 0.18 & 9.98 & 442.4 & 0.18 & 0.17 & 9.98 & 444.5 & 0.18 & 0.17 & 9.98 & 444.5 \\
\hline subthalamic nucleus & 0.05 & 0.04 & 18.6 & 560.6 & 0.07 & 0.05 & 18.39 & 560.7 & 0.07 & 0.05 & 18.48 & 561.6 \\
\hline thalamus & 0.01 & 0.00 & 3.88 & 262.8 & 0.05 & 0.02 & 3.81 & 261.4 & 0.00 & -0.02 & 3.90 & 265.8 \\
\hline precentral gyrus & 0.50 & 0.49 & 3.01 & 214.4 & 0.52 & 0.51 & 2.94 & 214.4 & 0.51 & 0.50 & 2.95 & 213.0 \\
\hline DLPFC & 0.39 & 0.39 & 2.50 & 179.6 & 0.40 & 0.39 & 2.49 & 180.6 & 0.40 & 0.39 & 2.49 & 180.5 \\
\hline MPFC & 0.53 & 0.53 & 2.29 & 162.5 & 0.54 & 0.53 & 2.27 & 163.3 & 0.54 & 0.53 & 2.27 & 163.4 \\
\hline Insular cortex & 0.57 & 0.56 & 2.86 & 205.2 & 0.58 & 0.57 & 2.83 & 205.2 & 0.58 & 0.57 & 2.83 & 205.3 \\
\hline postcentral gyrus & 0.27 & 0.26 & 3.987 & 267.8 & 0.27 & 0.26 & 3.98 & 270.0 & 0.27 & 0.26 & 3.98 & 270.0 \\
\hline precuneus & 0.28 & 0.27 & 4.20 & 278.1 & 0.28 & 0.27 & 4.20 & 280.3 & 0.28 & 0.27 & 4.20 & 280.3 \\
\hline Occipitotemporal cortex & 0.49 & 0.49 & 3.53 & 244.7 & 0.51 & 0.50 & 3.46 & 243.1 & 0.51 & 0.50 & 3.47 & 243.8 \\
\hline
\end{tabular}

Degrees of freedom = 93 for linear models and 92 for quadratic and exponential models.

Abbreviations: RMSE = root-mean-square deviation, AICc = Akaike information criterion with correction for small sample sizes; adj $=$ adjusted, DLPFC $=$ dorsolateral prefrontal cortex, MPFC $=$ medial prefrontal cortex 

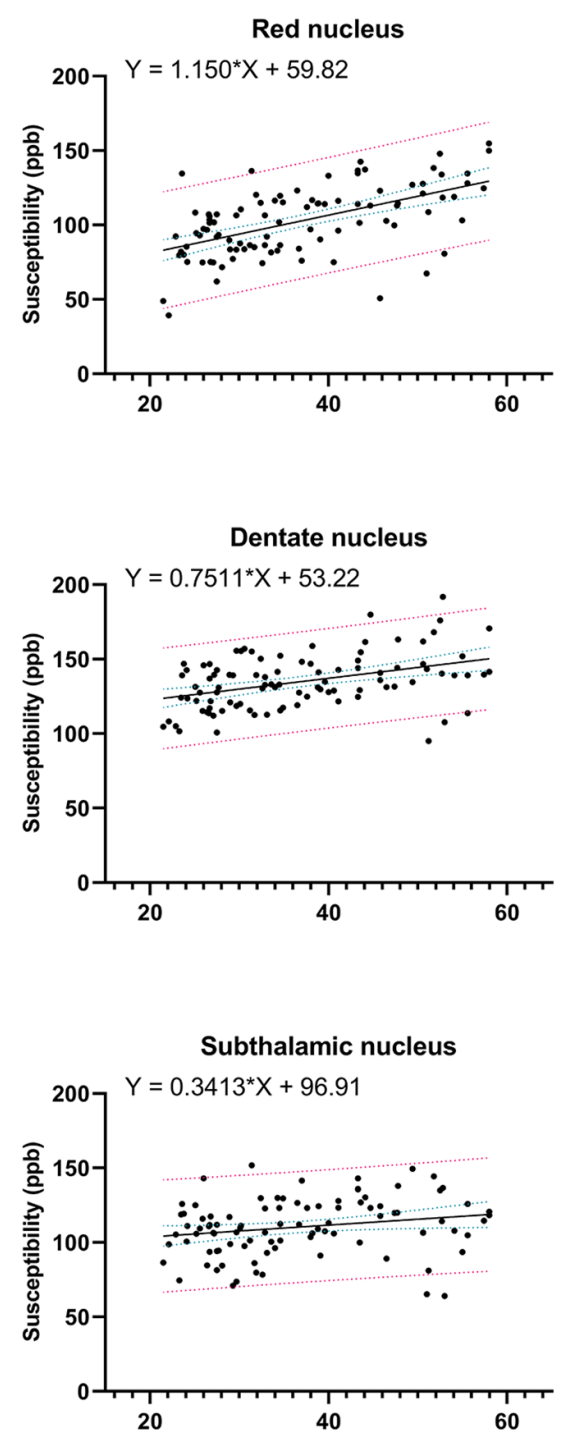

Thalamus

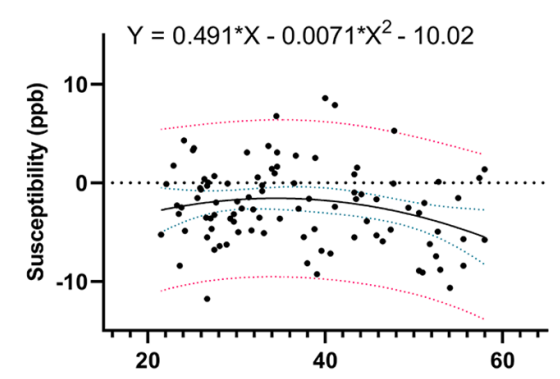

Putamen

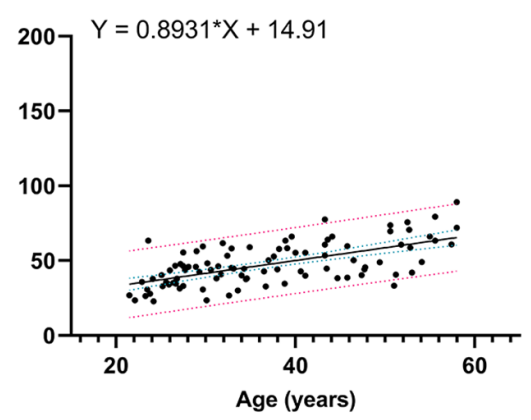

External globus pallidus

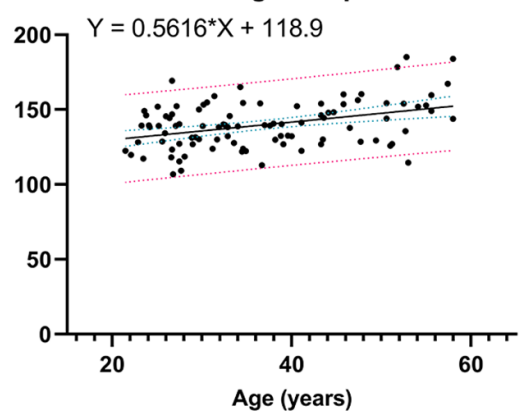

Internal globus pallidus

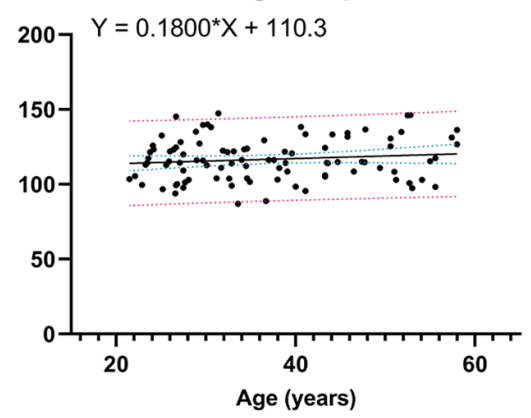

Pulvinar

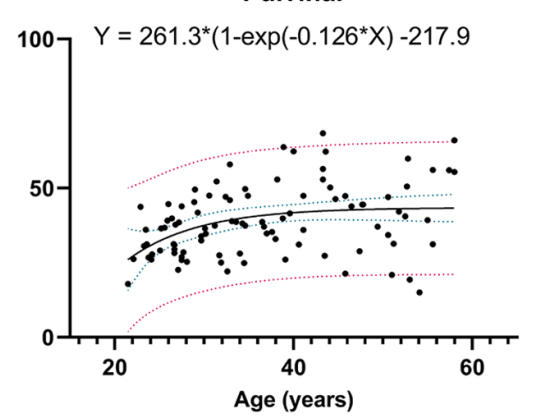

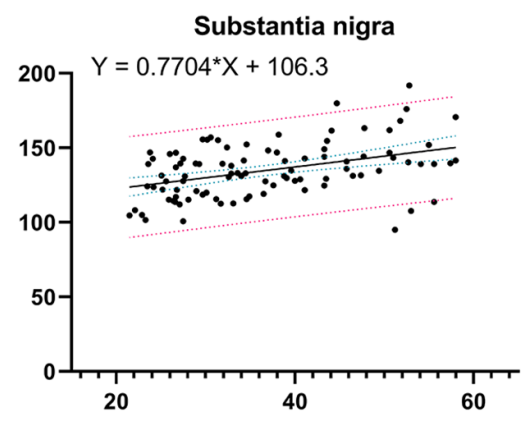

Caudate nucleus

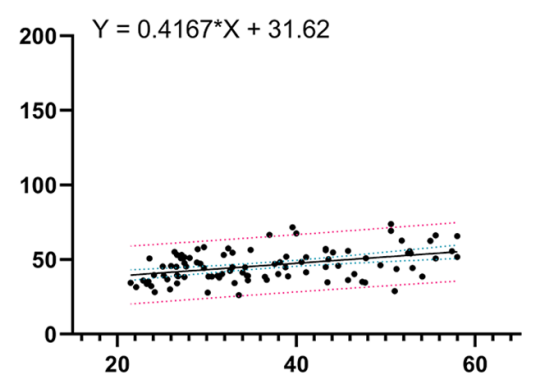

Figure S2 Corrected bulk magnetic susceptibility values (reference region = total white matter) in ppb in deep gray matter structures as a function of age with their respective regression fits and coefficients. Blue dotted lines represent $95 \%$ confidence bands of the regression fit and red dotted lines represent $95 \%$ population prediction bands. Please note different $\mathrm{Y}$-axis range for thalamus and pulvinar. Abbreviations: $\mathrm{ppb}=$ parts per billion; GP = globus pallidus; STN = subthalamic nucleus; n.a. = not available. 

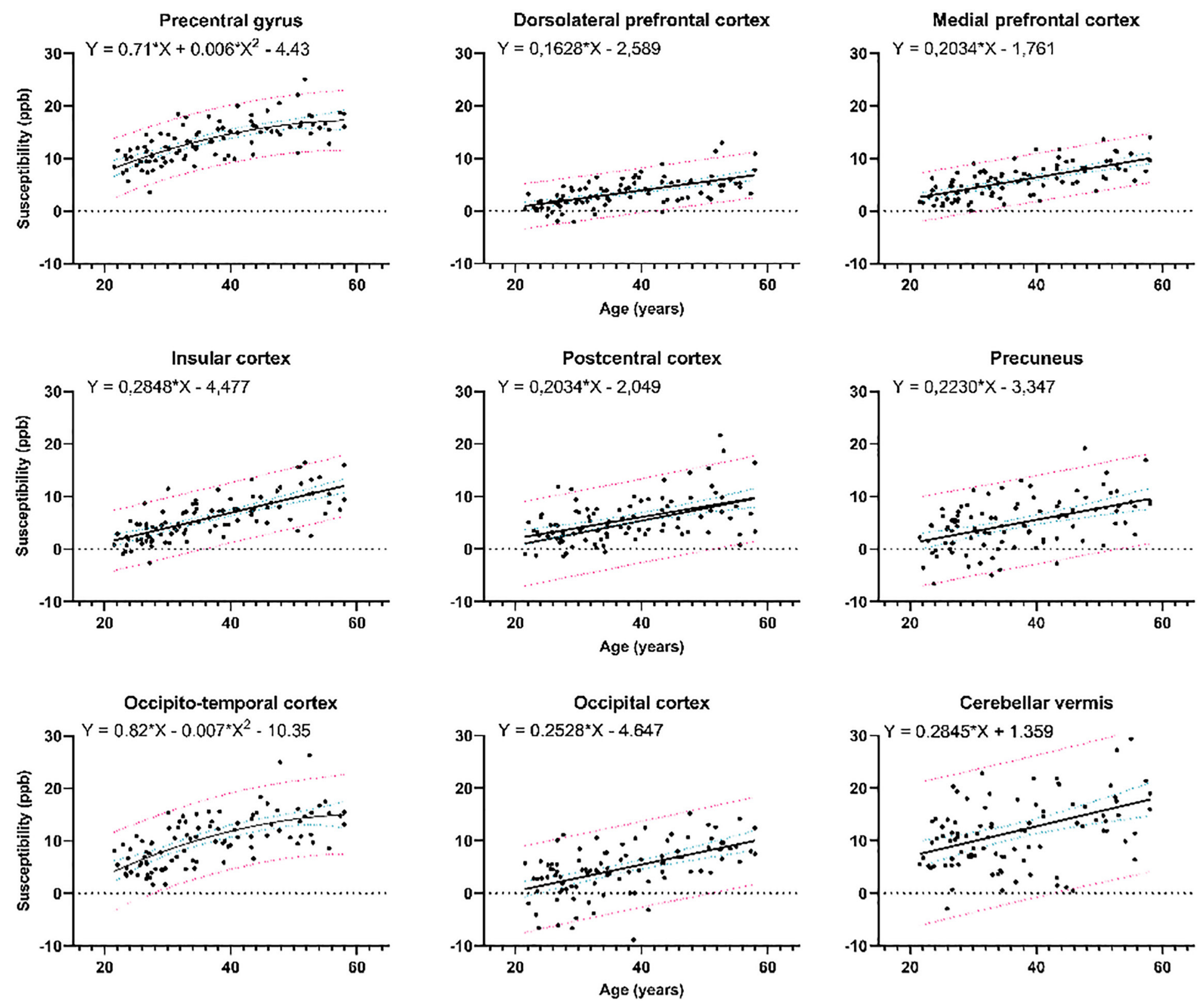

\begin{tabular}{|l|c|c|}
\hline & p-value & $\mathbf{R}^{2}$ \\
\hline Precentral & n.a. & 0.50 \\
\hline DLPFC & $<0.001$ & 0.39 \\
\hline MPFC & $<0.001$ & 0.46 \\
\hline Insular & $<0.001$ & 0.52 \\
\hline Postcentral & $<0.001$ & 0.24 \\
\hline Precuneus & $<0.001$ & 0.23 \\
\hline Occipito-temporal & n.a. & 0.45 \\
\hline Occipital & $<0.001$ & 0.29 \\
\hline Vermis & $<0.001$ & 0.16 \\
\hline
\end{tabular}

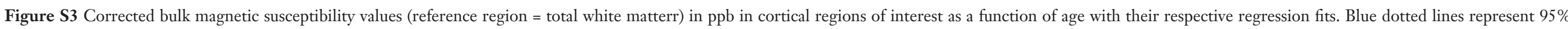
confidence bands of the regression fit and red dotted lines represent $95 \%$ population prediction bands.

Abbreviations: $\mathrm{ppb}=$ parts per billion; $\mathrm{DLPFC}=$ dorsolateral prefrontal cortex; $\mathrm{MPFC}=$ medial prefrontal cortex; $\mathrm{n} . \mathrm{a} .=$ not available 


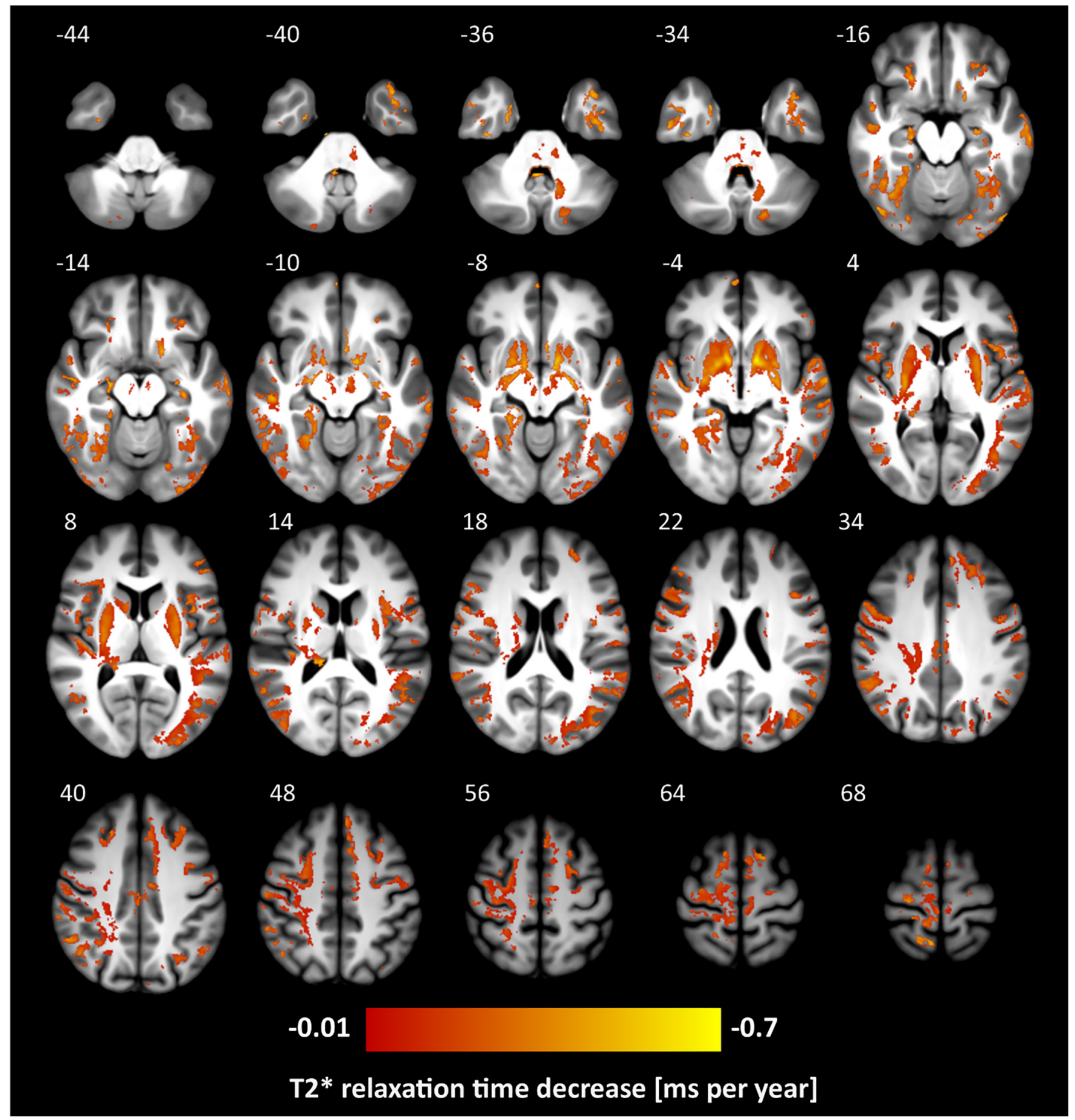

Figure S4 Statistical map of age-related $\mathrm{T} 2 *$ changes in the brain of normal adults represented as $\mathrm{T} 2{ }^{*}$ relaxation time decrease in ms per year (thresholded at $\mathrm{p}<0.05$ at cluster level and corrected for family-wise error; initial cluster definition threshold was set to $\mathrm{p}<0.005$ uncorrected). Z-coordinate in the MNI space in $[\mathrm{mm}]$ is indicated for each slice. 
Table S2 Clusters with significant age-related T2* relaxation time decrease identified by whole brain analysis with corresponding anatomical locations. Deep gray matter structures are in bold

\begin{tabular}{|c|c|c|c|c|c|c|c|}
\hline \multirow{2}{*}{$\begin{array}{l}\text { Cluster volume } \\
{\left[\mathrm{mm}^{3}\right]}\end{array}$} & \multirow{2}{*}{$\begin{array}{l}\text { Peak T2* relaxation } \\
\text { time change [ms/year] }\end{array}$} & \multirow{2}{*}{$\begin{array}{l}\text { Peak } \\
\text { T value }\end{array}$} & \multicolumn{3}{|c|}{ MNI coordinates $[\mathrm{mm}]$} & \multirow{2}{*}{ Peak Structure } & \multirow{2}{*}{ Anatomical structures within cluster } \\
\hline & & & $\mathrm{x}$ & $\mathrm{Y}$ & z & & \\
\hline 28449 & -0.7 & 6.5 & -28 & -34 & -24 & Inferior Temporal & Left Inferior, Middle, and Superior Temporal, Fusiform, Lateral, Middle, and Inferior Occipital gyri \\
\hline 52262 & -0.7 & 9.6 & 14 & -34 & -6 & Putamen & Right Putamen, Caudate, Globus Pallidus, Thalamic Pulvinar, Substantia nigra, Nucleus Ruber \\
\hline 8498 & -0.7 & 8.4 & -12 & 3 & -5 & Putamen & Left Putamen, Caudate, Globus Pallidus, Substantia nigra, Nucleus Ruber \\
\hline 191 & -0.7 & 4.9 & 31 & -36 & 0 & Hippocampus & Right Hippocampus \\
\hline 430 & -0.7 & 5.1 & -33 & -29 & -8 & Hippocampus & Left Hippocampus, Parahippocampal gyrus \\
\hline 760 & -0.6 & 4.3 & 36 & -77 & -18 & Lateral Occipital & Right Lateral Occipital, Inferior Occipital gyri, Occipital pole \\
\hline 527 & -0.6 & 6.2 & 48 & -24 & 17 & Posterior Insula & Right Posterior Insula, Supramarginal gyri \\
\hline 422 & -0.6 & 4.3 & 21 & -6 & -27 & Fusiform & Right Fusiform, Inferior Temporal, ParaHippocampal gyri \\
\hline 1051 & -0.6 & 5.9 & -3 & -15 & -19 & Pons & Pons, Mesencephalon \\
\hline 191 & -0.5 & 6.3 & -3 & 14 & -7 & Anterior Cingulum & Left Anterior Cingulum, Subcallosal gyri \\
\hline 1543 & -0.5 & 4.8 & 21 & 34 & -22 & OrbitoFrontal & Right OrbitoFrontal, Inferior Frontal gyri, Orbital, Anterior Rostral sulci \\
\hline 2186 & -0.5 & 5.0 & 67 & -39 & 2 & Superior Temporal & Right Superior, Middle, and Inferior Temporal, Middle and Inferior Occipital \\
\hline 1227 & -0.5 & 5.2 & 1 & -16 & 29 & Posterior Cingulate & Right Posterior and Middle Cingulate, Cingulate sulcus, Corpus callosum \\
\hline 572 & -0.5 & 4.8 & 31 & 29 & 2 & Anterior Insula & Right Anterior Insula, Inferior Frontal gyrus \\
\hline 3274 & -0.5 & 6.1 & -43 & 10 & 3 & Anterior Insula & Left Anterior Insula, Inferior Frontal, Precentral gyri, Rolandic Operculum \\
\hline 528 & -0.5 & 4.3 & 32 & -71 & 46 & Intraparietal sulcus & Right Intraparietal, Intraoccipital sulci, Middle Occipital gyrus \\
\hline 2405 & -0.5 & 5.5 & 53 & -51 & 42 & Angular & Right Angular, Supramarginal, Inferior Parietal, Superior Temporal gyri \\
\hline 322 & -0.5 & 4.4 & 8 & -53 & 68 & Precuneus & Right Precuneus, Parietal Superior, Cingulate gyri \\
\hline 1577 & -0.5 & 5.3 & -39 & 16 & -42 & Temporal Pole & Left Temporal pole, Inferior Temporal gyrus \\
\hline 10299 & -0.5 & 5.9 & -4 & 16 & 50 & Superior Frontal & Left Superior and Middle Frontal, Cingulate, Supplementary Motor area, Paracentral Lobule \\
\hline 359 & -0.5 & 5.5 & 7 & -6 & 50 & Cingulate sulcus & Right Cingulate, Paracentral Lobule \\
\hline 1950 & -0.5 & 5.1 & -59 & -15 & 37 & Rolandic sulcus & Left Rolandic, Precentral, Postcentral, Inferior and Middle Frontal \\
\hline 1379 & -0.4 & 4.5 & -53 & 34 & 5 & Inferior Frontal & Left Inferior Frontal \\
\hline 1392 & -0.4 & 4.8 & 49 & 24 & 20 & Inferior frontal & Right Inferior and Middle Frontal, Precentral sulcus \\
\hline 1433 & -0.4 & 5.2 & -21 & -79 & -16 & Left Lingual & Left Lingual, Calcarine gyri \\
\hline 500 & -0.4 & 5.5 & -18 & -76 & -35 & Cerebellum & Left Cerebellum \\
\hline 426 & -0.4 & 4.2 & -24 & 28 & -19 & Orbital sulcus & Left Orbital sulcus, Inferior Frontal gyrus \\
\hline 912 & -0.4 & 4.7 & 18 & -75 & -53 & Cerebellum & Right Cerebellum \\
\hline 644 & -0.4 & 5.2 & -25 & -63 & 42 & Intraparietal sulcus & Left Intraparietal, Parietooccipital sulci, Precuneus \\
\hline 522 & -0.3 & 4.7 & -13 & -56 & -36 & Dentate & Left Dentate \\
\hline 313 & -0.3 & 5.2 & -63 & -17 & 19 & Posterior Insula & Left Posterior Insula, Rolandic Operculum, Postcentral gyrus \\
\hline
\end{tabular}

